\title{
Use of INtelligent networks in the Universal Mobile Telecommunication System (UMTS)
}

\author{
Håkan Mitts \\ VTT Information technology, Telecommunication \\ P.O.Box 1202 \\ 02044 VTT \\ Finland \\ email: Hakan.Mitts@vtt.fi
}

\section{INTRODUCTION}

UMTS (Universal Mobile Telecommunication System) is a third generation mobile communication system currently being developed in Europe. UMTS related activities are lead by research conducted within the RACE II program and standardisation activities within the European Telecommunication Standards Institute (ETSI). ETSI SMG5 has the overall responsibility for UMTS related activities. More information on UMTS work in ETSI can be found in [SMG5]. For IN related matters also NA6 is involved. The ETSI working group responsible for Broadband ISDN, NA5, has created a special working group called Advanced Network Architectures (ANA). One of the objectives of ANA is to study UMTS impact on fixed networks.

Within RACE, the Mobile Project Line Assembly (MPLA), consisting of several RACE projects, is responsible for UMTS related activities. The major projects involved in UMTS development are MONET (R2066, responsible for fixed network aspects of UMTS including the relation between UMTS, IN and UPT), ATDMA (R2084, developing a radio interface for UMTS based on Time Division Multiple Access, TDMA), CODIT (R2020, developing a radio interface based on Code Division Multiple Access, CDMA), MBS (R2067, developing a broadband, $\sim 100 \mathrm{Mbit} / \mathrm{s}$, mobile access) and MAVT (R2072, studying aspects of a mobile audio and video terminal for UMTS). A RACE view of UMTS can be found in [BB92].

An effort similar to UMTS is underway in ITU under the name of FPLMTS (Future Public Land Mobile Telecommunication System), lately renamed to the more catchy IMT-2000 (International Mobile Telecommunication 2000). It is expected that UMTS and IMT-2000 will 
be compatible so as to provide global roaming but it is too early yet to say whether this goal will eventually be attained. ITU work on UMTS is described in [ITU].

This paper will present the use of Intelligent Networks in UMTS with an emphasis on the work done in RACE. To give some background, the UMTS system is first briefly described. Next the role and use of IN in UMTS are discussed. Finally some major areas will be presented, where significant enhancements to IN are expected.

\section{A BRIEF DESCRIPTION OF UMTS}

As for all mobile systems, UMTS provides wireless communication services to users on the move. The system supports roaming, that is the UMTS users can be reached and they can place calls where ever they are located within the coverage area of UMTS.

UMTS should be clearly distinguished from Universal Personal Telecommunication (UPT). UPT will offer discrete mobility, i.e. the possibility for a UPT user to register for incoming or outgoing services at any terminal. While moving between terminals, the UPT user is not registered and cannot therefore be reached. If a UPT user registers onto a wireless system like UMTS, the user also benefits from the wireless capabilities of the hosting system. One of the main design goals of UMTS is to allow UPT users to make use of UMTS in this way.

The distinction between UMTS and UPT has been made less clear by the fact that UMTS is expected to provide native support for UPT. Native support means that a UPT user (i.e. a user that only has a valid UPT subscription but with no UMTS subscription) could register onto a UMTS terminal without a prior subscription to UMTS. Such a mode of using UMTS would probably be more costly than using a normal UMTS subscription and thus UMTS users that are not UPT users will also exist. The resulting relation between UMTS and UPT, assuming native support for UPT in UMTS, is shown in figure 1 below.

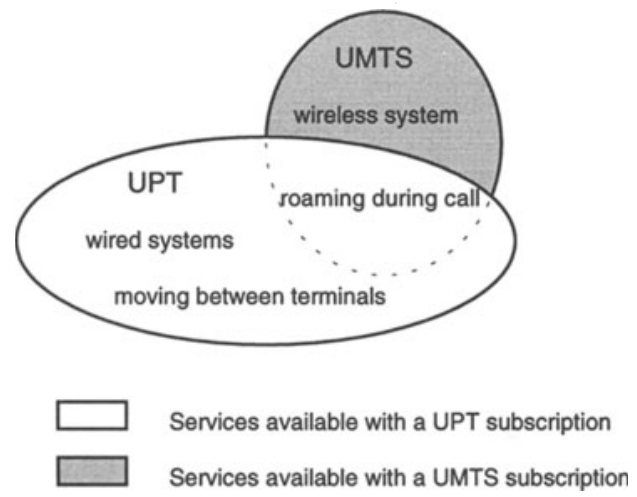

Figure 1. Relation between UMTS and UPT

UMTS is expected to satisfy a future demand for mobile multimedia services at transmission speeds up to a maximum of $2 \mathrm{Mbit} / \mathrm{s}$. The services will include high speed data services and video services like mobile videophony. The ability to provide mobile multimedia - and in particular video services - at an acceptable cost and with good quality is expected to be one of the main features distinguishing UMTS from second generation mobile systems like GSM. 
Other important features of UMTS will be the ability to support multicomponent calls as well as multiple simultaneous calls. As an example, a user might be engaged in a video conference that uses both a speech and a video component and at the same time initiate an interactive computer session from a personal computer connected to the UMTS terminal. In many respects the goals of UMTS are very similar to those of Broadband ISDN (B-ISDN) if one disregards the fact that the maximum transmission speed in UMTS will be much lower.

An other important aspect of UMTS is expected to be the support for various operating environments (public, domestic and business) using a single terminal. Global roaming between these environments will also be possible. To give an example, a user could use a UMTS mobile phone as a cordless phone at home. Next, without performing any explicit actions, the user could move outside the home to become reachable over the public network. Finally the user moves into an office and automatically becomes a wireless user of the PABX equipment in the office. It is even expected that the user could have maintained a call while moving from home through the public environment to the office.

\section{STRUCTURE OF THE UMTS NETWORK}

One of the main characteristics of UMTS is that it - in contrast to existing mobile systems like GSM - is planned for integration into a fixed network infrastructure and not as a separate overlay network. To achieve the integration, UMTS has been divided into three different parts as shown in figure 2 .

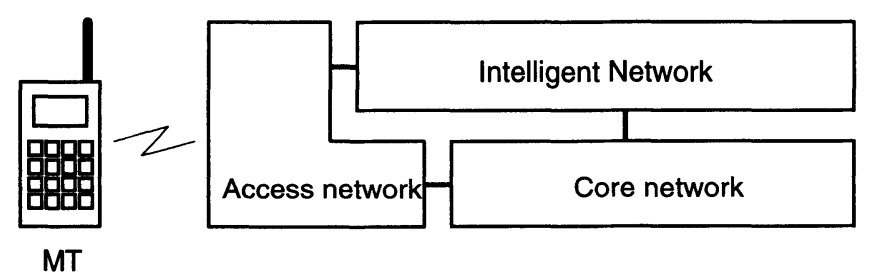

Figure 2. Main components of UMTS

The UMTS Access network is responsible mainly for radio related matters, it provides the radio access needed for wireless operation. Many important functions of a mobile system, e.g. handovers, are to a large extent implemented in the Access network.

The Core network provides the switching and transmission functions of UMTS. It will also provide interworking with fixed users and existing networks. Broadband ISDN is viewed as the likely candidate to act as the Core network of UMTS as it is the only fixed network technology that provides the required functions and the kind of flexibility needed to support the advanced UMTS functions.

Finally, the mobility specific control functions of UMTS are foreseen to be provided by an Intelligent Network (IN) based on the IN Capability Set 3 (CS-3) ${ }^{1}$. Some of the functions implemented using IN are:

1 IN Capability Set 2 is planned to support many of the functions required for UMTS but CS-3 is the first Capability Set that is expected to be able to support a fully functional UMTS network. 
- database functions to store and retrieve globally accessible UMTS data; most UMTS IN functions update or access this data

- location management functions needed to track the user while roaming

- user registration functions allowing users to register for services at different UMTS terminals

- security functions providing user identification and access control

- support for certain aspects of handovers, e.g. handovers occurring between Local Exchanges

The goal of UMTS development is that, of the three components above, only the Access network will be UMTS specific. To achieve this, a number of requirements on the reused components, i.e. the fixed network technology acting as the UMTS Core network and IN, have been identified. If these requirements are rolled into the base standards of the respective technologies - as currently seems possible - UMTS can be realised reusing major fixed network components to provide a highly integrated network supporting both fixed and mobile communication.

\section{THE ROLE OF IN IN UMTS}

Next we will examine in some detail how UMTS is expected to use IN. The functions needed for which UMTS is expected to use IN are very different from those targeted by IN CS-1. The main benefit of IN still remains the same in both cases: IN provides a separation between basic switching functions and the additional intelligence needed to support of mobile specific features.

This model is very useful for UMTS, as it offers a way to separate the UMTS specific control functions from the switching functions provided by the Core network. This in turn is essential to enable the (re)use of an existing fixed network switching fabric as the Core network of UMTS and thus fulfil the UMTS vision of a single, integrated network providing both fixed and mobile communication. It is an additional benefit that IN also could be used to provide value added services in UMTS. This could make interactions between value added services and mobility much easier to manage. The study of IN for UMTS has however largely disregarded this aspect of IN. As a consequence, this paper only discusses the use of IN to provide mobile functions to a fixed network.

To illustrate the use of IN for separating mobile functions from the functions of the Core network, the Network architecture of UMTS is shown in figure 3. Each node shown has been indicated as (mainly) performing fixed network switching functions, IN-type mobility related functions or radio access related functions. As can be seen, the architecture allows adding mobile functions to a fixed network by simply plugging in a number on IN nodes that support the mobile functions ${ }^{2}$. Figure 3 also shows the extent of the UMTS Access network.

2 Note that the Core network assumed here has been enhanced with some limited functions required to support the 'plug and play' mobility. 


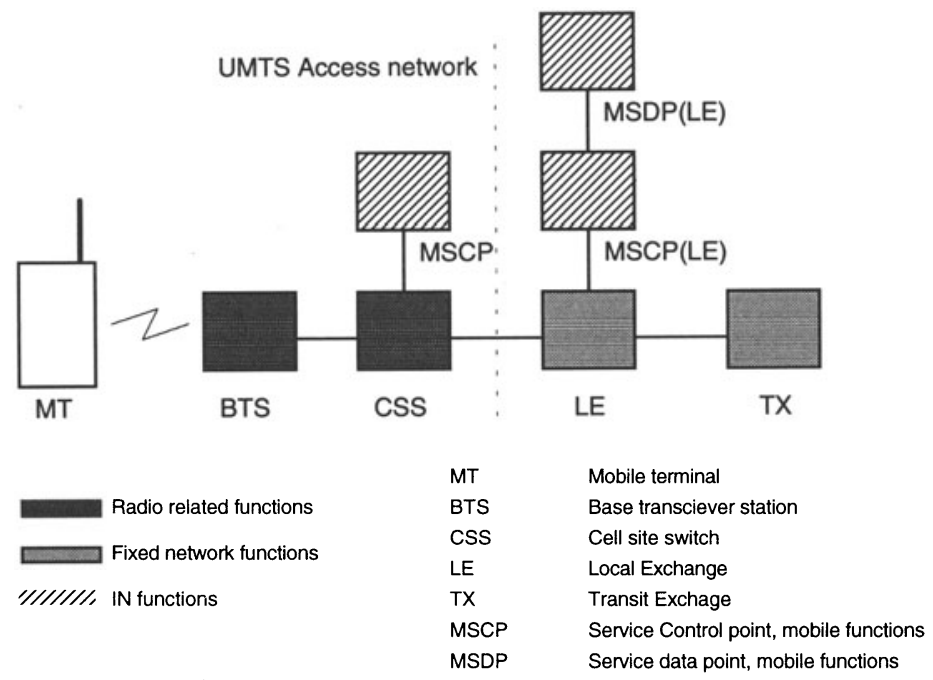

Figure 3. UMTS network architecture and major functions of the nodes

The MSCP is the UMTS node hosting the IN Service control function (SCF) with necessary provisions for the support of mobile users. Similarly, the MSDP is the UMTS node that hosts the IN Service data function (SDF). Figure 3 shows a slightly simplified view of UMTS in that the MSCP in the Access network could have an MSDP associated to it in large CPNs. Also the basic UMTS model identifies MSCPs and associated MSDPs controlling the Transit Exchanges but these have been omitted as they so far have very little functionality.

The UMTS network architecture shows maximal distribution in that the MSCP is assumed to host only the SCF-type functions of IN. Similarly the MSDP only contains the SDF functions. Nodes can of course be combined into bigger entities as required in an eventual implementation. It should also be reminded that the MSCP and MSDP are foreseen to be standard IN Entities and could therefore also be used to support any other IN-based services.

\section{UMTS REQUIREMENTS ON IN}

In this section we will identify a number of aspects of IN where improvements are critical to ensure the usability of IN for UMTS. Other requirements can be found in [BK\&94]. Requirements on B-ISDN can be found in [KMS94].

In its current form, CS-1 is mainly seen as a vehicle to quickly develop and deploy new value added services for the fixed telephone network, e.g. Freephone or Premium Rate services. This is in contrast to the mobile functions needed for UMTS that are well defined and will not change significantly over time. On the other hand, the performance requirements on the mobile related functions, and thus the IN components implementing them as well, will be very high and the distributed aspects of control will be much more pronounced than in CS- 1 . This implies that IN for UMTS will be a more stable but also more efficient system than IN CS-1 performing a well-defined task in an optimised way. This might eventually result in 
implementations where specialised IN nodes, responsible only for mobile functions, might occur.

\subsection{Distribution of UMTS functions}

Perhaps the main modification required to IN is the ability to support much more distributed operations. While current IN systems are based on a very centralised mode of operation, UMTS will require that various control functions are distributed so that they will reside close to the end user in order to provide adequate performance. This also implies that the same functions exist in a large number of UMTS/IN entities, each serving a small geographical area.

It is also foreseen that different UMTS/IN functions are distributed in different ways. Thus for one IN-based function, execution speed might not be very critical. This function could then be implemented in a fairly centralised way. Other functions might have much more pronounced efficiency requirements and might consequently be much more distributed. This is shown in figure 4 where the UMTS FunctionA is provided by a single MSCP for all areas shown while FunctionB is distributed and thus requires an MSCP for each area.

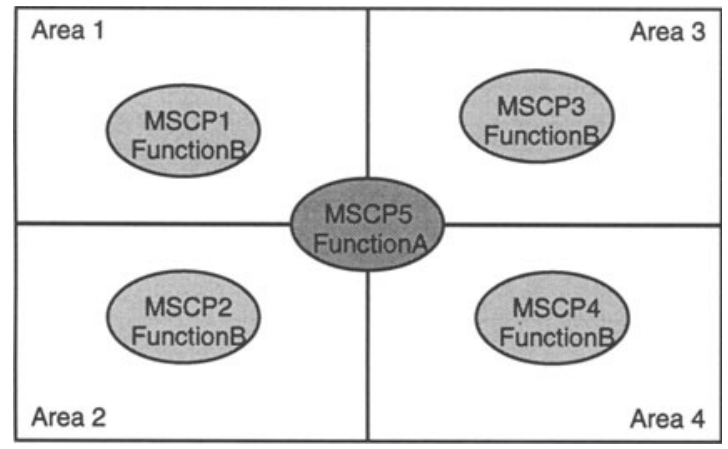

Figure 4. UMTS areas served by specialised MSCPs

Handovers (the actions required to support the movement of terminals when a call is active) bring yet other requirements on the distribution aspects of IN. As a terminal moves from one area to another, functions related to the terminal must be very quickly moved between entities responsible for the various areas visited. This implies a new, dynamic form of interaction between MSCPs not required by fixed network functions. This concept can be illustrated by assuming that a mobile moves from Area 1 to Area 2. If the mobile makes use of the UMTS/IN features implemented by FunctionB, the execution of the function needs to be moved from MSCP1 to MSCP2. This not only implies direct signalling between the MSCPS but also the existence of mechanisms to execute the transfer of control related to one mobile terminal between the MSCPs.

One final aspect of distribution is that IN functions are also foreseen to be moved to the UMTS access network (the MSCP connected to the CSS in figure 2) and onto customer premises. The interface between the UMTS LE (because the reuse of the B-ISDN LE is assumed) is the B-ISDN UNI including Q.2931, the B-ISDN UNI protocol, over a Signalling ATM Adaptation Layer (SAAL). This implies that the Signalling System 7, currently the main 
method of transporting signalling between IN entities in CS-1, is not available to interconnect the MSCPs in the access network to those deeper down in the network. This implies that IN must be updated to support new underlying signalling transport mechanisms in addition to Signalling System $7^{3}$.

Distributing IN functions to the access network also results in moving IN functions onto Customer Premises Networks (CPNs). When a CPN is used to provide UMTS services in either a domestic and business environment, the UMTS/IN functions pertaining to the access network actually reside in the CPN. Thus IN must also be updated to support interactions between customer operated IN entities and those of the UMTS network operators. This has implications mainly on security related matters in the communication between customer entities and network operator entities. Requirements related to authentication, etc. must be fulfilled to provide adequate security for the signalling functions when customer operated IN entities are involved.

\subsection{Handling of non-call related events}

Another important aspect of mobile systems that is not present in current IN specifications is the need to support events and actions taking place outside a call. Current IN systems only act on events initiated by triggers detected during the basic call processing in the SSP. This results in a tight coupling of the IN Basic Call Process (BCP) to all IN CS-1 functions.

In mobile system, including UMTS, many control procedures foreseen to use IN for their implementation take place outside a call. An example of such a function is Location updating whereby the location of a UMTS terminal is stored in the UMTS database to enable calls to be routed to the terminal as it moves. Location updates in UMTS can take place both during and between calls. Whenever a mobile terminal detects a change in its Location area (evident from data constantly broadcasted over the radio interface), the terminal reports its new location to the MSCP responsible for managing terminal locations in the new area. The MSCP then updates the information into the UMTS database (in the MSDP) to be globally accessible to any other entity that might require the information.

As Location updates a more frequent than calls, most Location updates take place when no call is present. It is certainly not acceptable to initiate a call just to execute the Location update mechanism, this would be far too costly in terms of overall system performance, in particular as the location update does not require any processing in the Local Exchange (LE).

Due to the above, it is not appropriate to model all IN related events in UMTS within a call state model. To support mobile systems, IN must be enhanced to support interactions between 'user entities' (here used in a very broad meaning) and IN entities without involving the basic call processing. To perform this efficiently requires that IN models and protocols be extended to provide direct 'user'-IN interactions. In this context both the UMTS mobile terminal and IN entities in the access network and CPNs could be viewed as users of the main IN functions located in conjunction with the UMTS Core network.

Recognising the problems above, a joint experts group was set up by ETSI SMG5 and NA6. Based on the work of this group, a new functional model for IN has been proposed. The 
functional model attempts to solve some of the problems related to non-call related functions [NA6]. The model is shown below in figure 5.

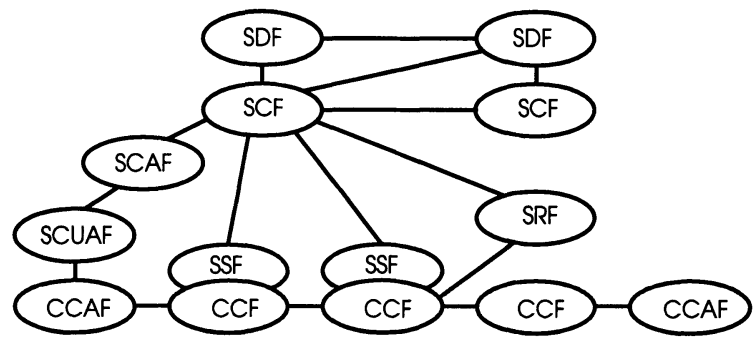

Figure 5. Proposed functional model for IN CS-2

In the model, two new Functional Entities are introduced, the Service Control Agent Functions (SCAF) and the Service Control User Agent Function (SCUAF). The SCUAF function provides service control access for users. It is the interface between user and network for service control. The SCAF provides the set of functions required for access and interaction between the SCUAF and a SCF. The SCAF and SCUAF are responsible for non-call related functions. They could well be used to model the non-call related functions required for UMTS. It should also be noted that the SCAF could be used to model the functions performed in the MSCP in the access network (see figure 2).

\subsection{Distributed database}

Data storage and retrieval are also a very important aspect of UMTS. While IN functions in support of IN CS-1 mainly read relatively static data from the IN database, UMTS functions frequently update the database as well. Further, access delay to the data is of crucial importance and the number of accesses per user will be very high. Further, due to the ability of users to roam, the data required to support a certain user might not be stored near the location where it is used.

To achieve the required processing speed for the UMTS data base and to manage the complexities related to locating the data for a given user, a number of mechanisms can be used. To maintain a simple interface between the UMTS entities using the data base and the data base itself, all UMTS data is stored in a single logical database distributed over all UMTS networks. The goal is to isolate the UMTS entities processing the data from the complexities related to the database implementations. This allows UMTS functions to access the required data without the need to know details related to the data base like the current location of the data for a given user. It also allows the data base to be modified and improved without changing the way it is used.

As a consequence, even though the MSDPs are shown as separate entities, they all provide access to a single global database. The main functions of this database are:

- the basic functions of providing read and write access to the data

- locate the requested data within the database

- move data to locations close to the user when the user has roamed to provide fast access to the data where it is frequently being used 
- provide mechanisms that ensure the integrity and reliability of the data even when it is copied between locations and possibly duplicated as a consequence

- ensure that the data is only access by entities that have been authorised to do so

Many aspects of the UMTS database can be found in the X.500 specification. X.500 has also been used as the basis when defining the improved access interface between SCF and SDF in the ETSI Core INAP specification [Core]. It therefore seems likely that the UMTS distributed database will resemble the X.500 database even though X.500 cannot directly be used.

\section{CONCLUSIONS}

Evidently, due to the gap between IN CS-1 and the requirements imposed on IN by UMTS, a number of modifications and improvements to IN are necessary before UMTS can be implemented as planned. If these enhancements are rolled into standard IN - as now seems to be possible mainly in the CS-3 time frame - standard IN can be used to support mobile control functions in UMTS and thus fulfil the goal of reusing existing systems to implement UMTS.

As significant changes to IN are required, one can ask whether it has been correct to choose IN as the starting point for UMTS developments. Perhaps a better and more economic solution could have been found by starting from a clean slate? While this question certainly is valid, the advantages of the chosen path must not be forgotten. At the time when UMTS developments where started, no other scheme existed that provided the crucial division between basic (fixed network) switching functionality and the processing intensive functions to be added to provide the mobile functions.

Using IN is also an important step in enabling the integration between UPT and UMTS. As UPT will use IN, integrating the two technologies is expected to be much easier when UMTS is based on IN. If the current vision of enabling the use of UMTS terminals with only a UPT subscription becomes reality, mobile communications have taken a great step forward towards enabling ubiquitous communication.

It should also be pointed out that even though a number of requirements stemming from UMTS have been pointed out, they are not unique to UMTS. For instance UPT has many similar requirements, as have other advanced functions. By looking to all supported services in an integrated way, duplication of functionality and effort can certainly be avoided.

Finally it should be mentioned that other recent developments to provide advanced service processing separate from the basic switching functions, notably the TINA (Telecommunication Information Network Architecture) initiative [Nat93], have goals that are well aligned with the long term goals of IN. Thus basing UMTS development on IN is not in contradiction with other emerging technologies but evidently efforts are needed to realise a merging if it becomes desirable.

\section{ACKNOWLEDGEMENTS}

This paper is based on work done in the RACE II project MONET (R2066) where the author is working. The views presented are however those of the author and do not necessarily represent those of the project as a whole. The work has been partly funded by the Technology Development Centre in Finland (TEKES). 


\section{REFERENCES}

[BB92] Hans de Boer and Evert Buitenwerf, Networks for 3rd generation mobile telecommunication systems, Proceedings 1992 IEEE International Conference on selected topics in wireless communications, 25-26 June, 1992, Vancouver.

[BK\&94] Wouter van den Broek, Eric Kuisch, Chris van Maastricht, Toon Norp, Impact of UMTS on IN Developments, $3^{\text {rd }}$ International Conference on Intelligence in Networks, Bourdeaux, France, October 11-13, 1994 (to be published)

[Core] ETSI ETS 300374-1, Core Intelligent Network Application Protocol (INAP), 1994, European Telecommunications Standards Institute, Sophia Antipolis, France.

[ITU] ITU-RS TG8/1, FPLMTS - Future Public Land Mobile Telecommunication Systems, ITU document 8-1/TEMP/173.

[KMS94] John Korinthios, Håkan Mitts, Efstathios Sykas, Scenarios for integration of the Universal Mobile Telecommunication System (UMTS) into Broadband ISDN, 44th Vehicular Technology Conference, Stockholm, Sweden, June 7-11, 1994.

[NA6] ETSI NA6, Enhancements of the IN distributed functional architecture, TC-TR NA 60401, Version 2.0.0, European Telecommunications Standards Institute, Sophia Antipolis, France.

[Nat93] N. Natarjan, M. Ferro The Distributed Processing Framework in the INA Architecture, The 4th TINA workshop (TINA'93), September 27-30, 1993, L'Aguila, Italy.

[SMG5] ETSI SMG5, Work programme for the standardization of the Universal Mobile Telecommunications System (UMTS), DTR/SMG-05 00-01, European Telecommunications Standards Institute, Sophia Antipolis, France. 